\title{
Analysis and Design of the Logistics System for Rope Manufacturing Plant
}

\author{
Xue Sun $^{1,2, *}$, Chao-Chin $\mathrm{Wu}^{3}$, and Liang-Rui Chen ${ }^{2}$ \\ ${ }^{1}$ College of Urban Rail Transit and Logistics, Beijing Union University, Beijing, China \\ 2 Department of Electrical Engineering, National Changhua University of Education, Changhua, Taiwan \\ ${ }^{3}$ Department of Computer Science and Information Engineering, National Changhua University of Education, Changhua, Taiwan
}

\begin{abstract}
In order to promote logistics system for manufacturing plant, this paper proposed a new design for the logistics system of a rope manufacturing plant. Through the analysis in the aspects of workshop facility layout, material handling and inventory management, the original logistics system of the plant is optimized. According to the comparison of the simulation results between original and optimized design, the optimized model has the higher productive efficiency. This can provide the references for the other manufacturing plant in analysis and design of the logistics system to improve plant efficiency.
\end{abstract}

\section{Introduction}

With the rapid development of technologies, in manufacturing industry original single variety of mass production is transformed into low-volume and multispecies production. The new production method is more adaptable to the faster changes of the market. In the process of manufacturing, it spends more than $90 \%$ of the time in the logistics process such as transport, handling, and packaging. However, some manufacturing enterprises especially small and medium enterprises only perform simple analysis by experience of practice, which results in contradiction between the backward enterprise logistics system and improvement of production becoming increasingly prominent. Therefore, making a design of good internal logistics system can not only reduce the production costs, but also help enterprises to improve production efficiency, thereby enhance the competitiveness of enterprises.

This paper takes a certain rope manufacturing plant as the research object. As it is a new plant, the entire logistics systems within the plant have a lot of imperfections. According to the investigation of the plant, there are unreasonable facility layout of the production, not high efficiency of logistics handling, low space utilization and other issues. This research is to analyze and optimize the plant logistics system on workshop facility layout, handling and inventory management. Finally, the rational design of the optimization system is verified by simulation.

\section{Factory logistics principle}

Factory logistics is from the beginning of raw materials procurement into the plant. After certain manufacturing processes, formulate the semi-finished products, and then assemble them into a certain value of the finished products. Ultimately store the finished products in the warehouse or transport them to the users. In this process, materials have experienced a series of circulation from the procurement into the storage to manufacturing and finished goods shipping. Generally speaking, the factory logistics refers to the flow of goods within the factory.

The main research to factory logistics system is as follows:

(1) Survey data collection

Collect the raw data of the plant in a systematic way, including production operations and related data, to analyze the existing problems of the plant.

(2) Plant facility layout

Facility layout looks at physical allocation of space for economic activities in the plant. The main objective of the facility layout planning is to design effective workflow as to make equipments and workers more productive.

(3) Material handling

Material handling in the plant mainly involves shortdistance movement within the confines of a building or between a building and a transportation vehicle [1]. Material handling also affects facility layout to some extent, and the layout can be modified according to the efficiency of the material handling.

(4) Warehouse and inventory management

Warehouse and inventory management in a plant is the practice overseeing and controlling of the ordering, storage and use of components that a manufacturing enterprise uses in the production of the items it sells.

In the following sections, we choose a manufacturing plant called Plant L, which produces ropes for hunting, carrying, lifting and climbing, to analyze and design the plant logistics system in plant facility layout, material handling system and inventory management. 


\section{Facility layout of rope manufacturing plant}

Plant L is a high-tech manufacturing enterprise which is mainly produces many kinds of ropes. This plant has a better sales performance, that the monthly total sales of various products are generally around one hundred tons. According to the actual investigation, the plant facility layout was almost depended on experiences of managers and there was no systematic analysis, resulting in low logistics efficiency and not high space utilization. To solve the problems, we adopted Systematic Layout Planning (SLP) to analyze and design the plant facility layout. Through systematic analysis of SLP, we got three alternative options. Furthermore we used Analytic Hierarchy Process (AHP) to select the best scheme.

\subsection{Overview of SLP}

The SLP is a tool used to arrange a workplace in a plant by locating areas with high frequency and logical relationships close to each other [2,3]. There are four levels of detail in plant layout design what are site layout, block layout, detailed layout and workstation layout. Five major elements of a SLP methodology are P (Products), Q (Quantity), R (Routing), S (Service), and T (Timing). The basic steps of SLP are:

- Prepare original data

The preparation of the original data includes clarification of five major elements and division of the plant operating units. This information is the prerequisite and key for system layout design.

- Analyze logistics relationship

Logistics analysis is an important part of the facility layout. According to process flow diagram, some factors about logistics between two departments such as flow costs can be quantified. Such relationships can be described by using REL diagrams.

- Analyze non-logistics relationship

Non-logistics relationship is the key element of facility layout design. The factors of non-logistics are such as noise, safety and pollution, the continuity of the production process, and convenience of material handling. The non-logistics relationship between two departments is also quantified by using REL diagrams.

- Analyze comprehensive correlation and draw related table

In the actual, score of comprehensive correlation can be obtained by the weighting of logistics analysis and non-logistics analysis, and then comprehensive correlation table can be drawn.

- Draw space relationship diagram

In this part, substitute in the actual area on each department and fit the departments into the available space.

- Put in the constrains

Finally, other existing constrains are employed to cut down number of feasible solutions, to result in a small set of solutions. From among these, direct comparison can be used to rank, eliminate, or select optimum design.

\subsection{Application of SLP in Plant L}

There are mainly nine raw materials which can be divided into two categories, one need for drawbench and the other need not. According to the collected information, daily demand of raw materials can be obtained which is shown in Table 1. The production process of Plant L includes inspecting, wire drawing, twisted strand, stranded rope, and packaging. Semiproductions produced by wire drawing, twisted strand and stranded rope must be inspected in order to ensure the product quality. Draw the process flow diagram for plant L is shown in Fig.1. Ten departments are separated, and the required area for each department is calculated and presented in Table 2.

Table 1. Daily demand of raw materials.

\begin{tabular}{|c|c|c|}
\hline ID & Raw material name & Weight(kg/day) \\
\hline 1 & Polyester fiber & 1360 \\
\hline 2 & Polypropylene fiber & 1190 \\
\hline 3 & Nylon & 170 \\
\hline 4 & HMPE & 170 \\
\hline 5 & Sisal & 136 \\
\hline 6 & Cotton & 272 \\
\hline 7 & Kevlar fiber & 103 \\
\hline 8 & PP & 933 \\
\hline 9 & PE particle & 234 \\
\hline
\end{tabular}

Table 2. List of department and required space.

\begin{tabular}{lcc}
\hline No. & Department & Size $\mathrm{m}^{2}$ \\
\hline 1 & Raw Material Storage & 795 \\
2 & Inspecting Department & 270 \\
3 & Wire Drawing Department & 2072 \\
4 & Twisted Strand Department & 5325 \\
5 & Strand Rope Department & 2560 \\
6 & Waste Department & 135 \\
7 & Semi-product Department & 576 \\
8 & Finished Product Department & 672 \\
9 & Packaging Department & 784 \\
10 & Office Department & 135 \\
\hline
\end{tabular}

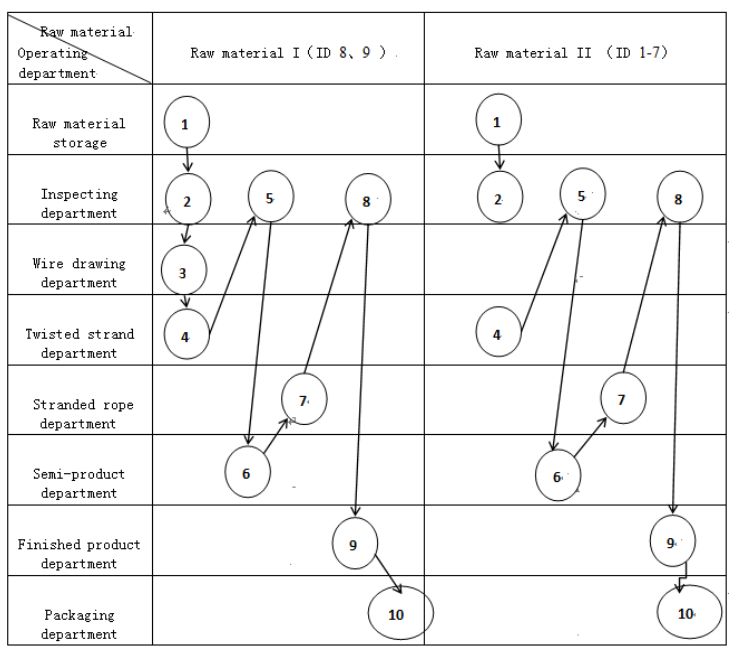

Fig. 1.Process flow diagram. 
In SLP technique, to the rope plant L, after analysis of logistics and non-logistics, REL chart is required to show the importance of adjacency between pair of departments. Fig. 2 is the REL chart where have A, E, I, $\mathrm{O}, \mathrm{U}$ to represent absolutely necessary, especially important, important, ordinary closeness okay, unimportant, and undesirable. REL chart is used to develop the relationship diagram shown in Fig.3, which has three different alternatives to be generated for plant L.

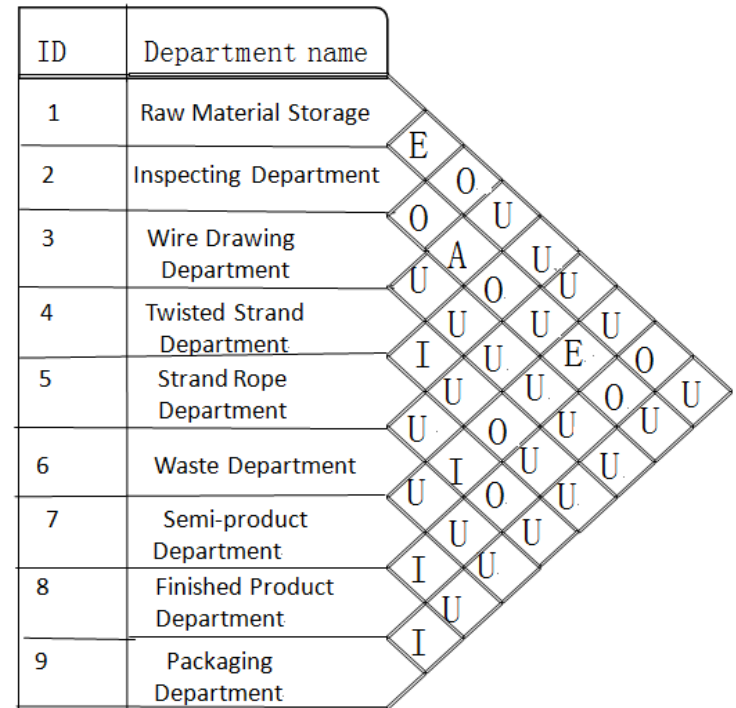

Fig. 2. REL chart.

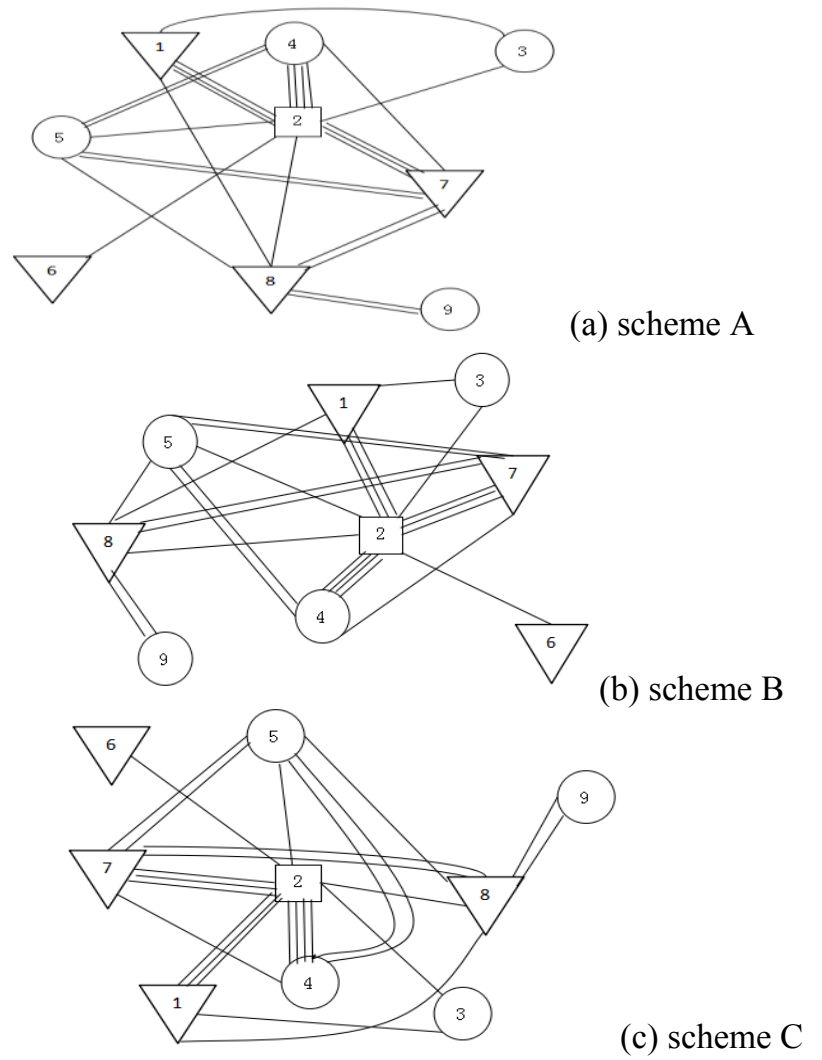

Fig. 3. Relationship diagrams.

\subsection{Evaluate alternatives for Plant $L$ with AHP method}

Three alternatives of space relationship diagrams are produced by SLP method. One of the best among them should be obtained as a decision. The analytic hierarchy process (AHP) method can be used. AHP method is a structured technique for analyzing and organizing complex decisions based on psychology and mathematics [4]. In accordance with the production status of L plant, five evaluation factors were chosen for study, what are safety and comfort of the working environment, the space utilization in the workshop, the convenience of the management, the efficiency of production logistics and the convenience to the warehouse.

Let $U$ be the five evaluation factors, and set $V$ as the three types of the relationships:

$U=\{$ safety and comfort of the working environment $(u 1)$, the space utilization in the workshop $(u 2)$,

the convenience of management ( $u 3)$,

the efficiency of production logistics $(u 4)$,

the convenience to the warehouse $(u 5)\}$ And

$$
V=\{\operatorname{REL} 1(v 1), \operatorname{REL} 2(v 2), \operatorname{REL} 3(v 3)\}
$$

Based on the enterprise managers and experts scoring for statistics to form the single factor evaluation matrix, collect the scoring and statistics and use formula for processing the statistical results. Through the test of consistency of judgement matrix, get the weights of the influence factors as:

$\omega=\left(\begin{array}{lllll}0.114206 & 0.126946 & 0.097889 & 0.370940 & 0.290019\end{array}\right)^{T}$

And then get the results of comprehensive evaluation is:

$$
\left[\begin{array}{lll}
\omega A & \omega B & \omega C
\end{array}\right]=\left[\begin{array}{lll}
0.1897 & 0.2360 & 0.5743
\end{array}\right] .
$$

Because the weight value accounted for 0.5473 is the largest, the plant $\mathrm{L}$ should choose the scheme $C$ of the space relationship digrams. The facility layout for plant $\mathrm{L}$ is shown in Fig.4.

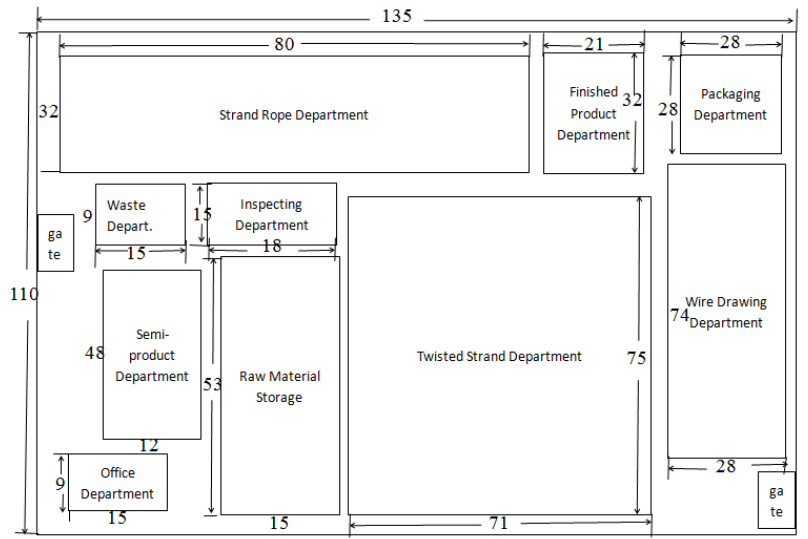

Fig. 4. Facility layout of plant L. 


\section{Analysis of material handling system}

Material handling is the movement, protection, storage and control of materials and products throughout manufacturing, warehousing, distribution, consumption and disposal. Material handling incorporates a wide range of manual, semi-automated and automated equipment and systems that support logistics and make the supply chain work.

For plant L, after analysis and design of the facility layout, the handling analysis can be carried out in conjunction with F-D diagram. Calculate the distance between the pairs of the department having the handling contact, and draw the F-D diagram shown in Fig.5.

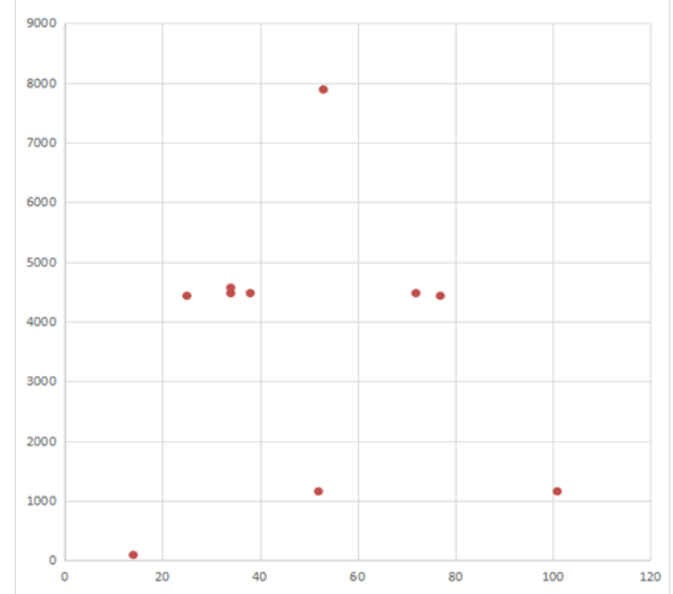

Fig. 5. F-D diagram for plant L

From the F-D diagram for plant L, most of the handling is concentrated in the lower part of the figure which is for a reasonable handling. The main handling tools of plant $\mathrm{L}$ are forklifts. It is suitable for the handling needs. The scheme $C$ of the layout of the handling for plant $\mathrm{L}$ is feasible.

\section{Analysis of inventory management system}

In manufacturing enterprises, good inventory management is critical in ensuring the normal production and the demand. $\mathrm{ABC}$ classification is an effective method for managing the products. $\mathrm{ABC}$ analysis is also called Pareto analysis, which is a method of classifying events, items, or activities according to their relative importance [5].

Through the investigation of the plant $\mathrm{L}$, there are about thirty kinds of products, involving a number of application areas, such as outdoor leisure, rescue, protection, safety, fishery, yachts, agriculature, animal husbandry, garden, and other daily necessities. We have $\mathrm{ABC}$ classification for the products, the specific steps are:
(1) According to the product price and inventory level to caculate monthly inventory amount.

(2) Order the monthly inventory amount from high to low order of the products.

(3) Calculated the accumulated monthly inventory based on the order of the products after sorting.

(4) Calculate the percentage of accumulated monthly inventory for each kind of product and classify it by $\mathrm{ABC}$ classification.

After the steps, products can be divided into three classification. Products in class A need to be focused on management, the raw materials for this type of product are regularly ordered and ordering cycle should be minimized. Class B is in general management in the use of quantitative orders, which adjusts the inventory level according to the business strategy. Class $\mathrm{C}$ products can be mass-produced due to the small amount of captial occupied.

\section{Modelling and simulation}

We adopted simulation software Flexsim 7.5 to model and simulate our analysis and design which we called optimized workshop. Build up Flexsim model for original and optimized layout of plant $\mathrm{L}$ according to our analysis and design. Set the simulation runtime of the working time that is eight hours or 28800 seconds a day. The summary reports of simulation results are shown in Figure 6 and Figure 7. Figure 6 is a report for original layout, and Figure 7 is a report for optimized layout.

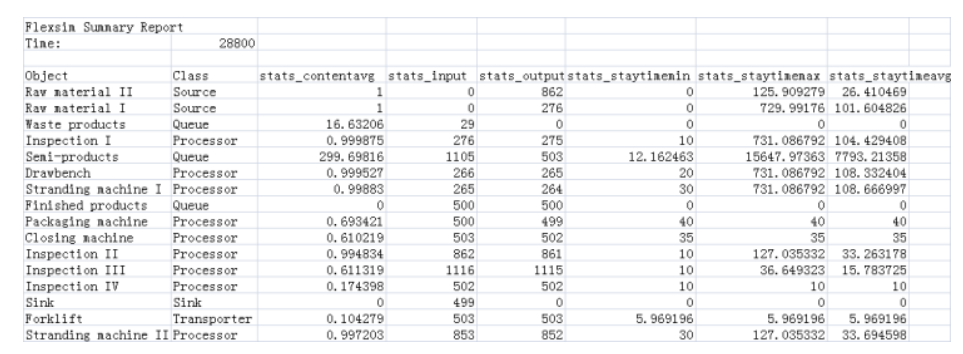

Fig. 6. A report for original layout

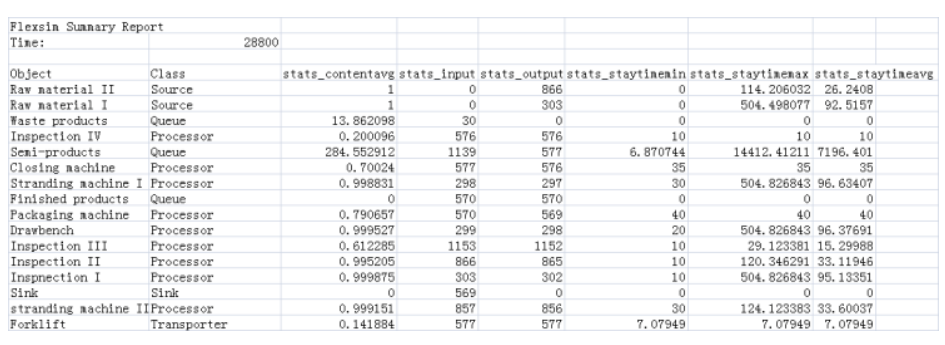

Fig. 7. A report for optimized layout

From the comparison of the two reports, for the semifinished products, the capacity of the optimized workshop is smaller than that of the original workshop. Optimized workshop can save the space area of semifinished products, and the waiting time of them for processing is shorter, further to reduce the waste of time. 
Overall, the sum of the average staytime of the optimized workshop is shorter than that of original workshop, that indicates the handling efficiency of the system is improved. In the same period of time, outputs of the optimized workshop are 569, which is significantly higher than the outputs of the original workshop 499. The productive efficiency is increased by nearly $14 \%$. Therefore, the optimized solution (Scheme C) of the logisitics system for plant L is better than the original design.

\section{Conclusions}

The purpose of this paper is to develop a new design for analyzing and designing of the logistics system for manufacturing plant. This research took a certain rope manufacturing plant as an example, through analysis of current production and logistics in the plant, provide the logistics design in workshop facility layout, material handling and inventory management. For workshop facility layout, SLP and AHP method can be combined for the best layout selection. With F-D diagram, analyze the material handling to adjust the workshop layout. For inventory management, use $\mathrm{ABC}$ classification to analyze the plant inventory. Finally, compare the optimized design to the original design with the simulation software. The results indicate our design of logistics system for the plant is better than the original design, and it can improve the production efficiency of the plant.

This work was supported by the 2017 Project supported by China Society of Logistics under Grant No. 2017CSLKT3-163.

\section{References}

1. Coyle, J.J., "Management of Business Logistics", Mason, OH: South-Western. p. 308 (1992)

2. Muther, R., "Systematic layout planning", Cahners Books (1984)

3. Ojaghi Y, Khademi A, Yusof $N$, et al, "Production Layout Optimization for Small and Medium Scale Food Industry", Procedia Cirp, 26:247-251 (2015)

4. Sun X, "E-Commerce Logistics Distribution Mode Analysis and Decision", Applied Mechanics \& Materials, 744-746:1873-1877 (2015)

5. Sun X, "Simulations on the Spare Parts Inventory Management of Equipment Maintenance in Manufacturing Enterprise Based on Flexsim", Advanced Materials Research, 715:3181-3186 (2013) 